\title{
Optical Trapping at Gigapascal Pressures
}

\author{
Richard W. Bowman, ${ }^{*}$ Graham M. Gibson, and Miles J. Padgett ${ }^{\dagger}$ \\ SUPA, School of Physics and Astronomy, University of Glasgow, Glasgow G12 8QQ, United Kingdom
}

Filippo Saglimbeni and Roberto Di Leonardo

IPCF-CNR and Dipartimento di Fisica, Università di Roma "La Sapienza", Piazzale Aldo Moro, 2, 00185 Roma, Italy

(Received 8 November 2012; published 28 February 2013)

\begin{abstract}
Diamond anvil cells allow the behavior of materials to be studied at pressures up to hundreds of gigapascals in a small and convenient instrument. However, physical access to the sample is impossible once it is pressurized. We show that optical tweezers can be used to hold and manipulate particles in such a cell, confining micron-sized transparent beads in the focus of a laser beam. Here, we use a modified optical tweezers geometry, allowing us to trap through an objective lens with a higher working distance, overcoming the constraints imposed by the limited angular acceptance of the anvil cell. We demonstrate the effectiveness of the technique by measuring water's viscosity at pressures of up to $1.3 \mathrm{GPa}$. In contrast to previous viscosity measurements in anvil cells, our technique measures absolute viscosity and does not require scaling to the accepted value at atmospheric pressure. This method could also measure the frequency dependence of viscosity as well as being sensitive to anisotropy in the medium's viscosity.
\end{abstract}

DOI: 10.1103/PhysRevLett.110.095902

PACS numbers: 66.20.Ej, 07.35.+k, 62.50.-p, 87.80.Cc

Introduction.-Since the pioneering work by Bridgman [1], high pressure has been shown to be capable of inducing remarkable structural changes in soft and biological matter. Diamond anvil cells (DACs) permit a small sample of material to be compressed to pressures up to hundreds of gigapascals [2,3] while, simultaneously, probing it with radiation through the diamond anvil windows. For this reason, optical spectroscopy and x-ray diffraction have been so far the primary tools of investigation while mechanical and rheological measurements, requiring the direct and local application of forces, have posed significant challenges [4]. Optical tweezers, however, allow us to trap and manipulate multiple objects in three dimensions using controlled and calibrated forces exerted by tightly focused laser beams [5]. Tightly focused light requires the use of microscope objectives having a high numerical aperture (NA) and a short working distance, a requirement that seems to make this technology incompatible with standard DAC. On the other hand, counterpropagating optical traps, which predate the more widely used single beam tweezers [6], can be implemented at lower NA, allowing the use of lower magnifications and longer working distances [7]. This enables the use of sample volumes much larger than the $\sim 100 \mu \mathrm{m}$ normally accessible to optical tweezers [8]. Recently, counterpropagating optical traps have been implemented with a single microscope objective and a mirror behind the sample $[9,10]$ to form

Published by the American Physical Society under the terms of the Creative Commons Attribution 3.0 License. Further distribution of this work must maintain attribution to the author(s) and the published article's title, journal citation, and DOI. the backwards-propagating beam. Creating two foci, one in front of and one behind the mirror as shown in Fig. 1, forms a counterpropagating trap as the focus behind the mirror is reflected back into the sample. Such manipulations can be easily accomplished using a spatial light modulator (SLM) as found in holographic optical tweezers [11,12]; indeed, we can create arbitrary 3D configurations of laser foci, corresponding to many independent optical traps in three dimensions. As optical traps thus formed can trap larger objects over larger volumes, they have been dubbed

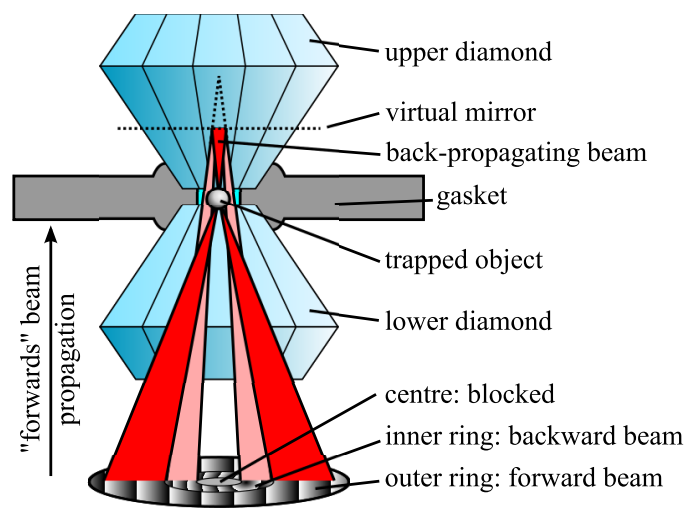

FIG. 1 (color online). We form an optical trap inside a diamond anvil cell by forming several beams using an SLM such that the object is held between two counterpropagating laser foci. The SLM splits the beam in the pupil plane of a microscope objective, such that the most highly converging rays form the forward-propagating focus and the middle of the pupil plane is used for the backwards-propagating beam. The central 1/10 of the beam is blocked to prevent out-of-focus light interfering with the trap. 
"macro-tweezers" [13]. Here, we demonstrate that, using a holographic counterpropagating trap, it is possible to use optical manipulation to probe the mechanical properties of high pressure samples contained in a diamond anvil cell. We describe the implementation of this technique on a portable holographic optical tweezers instrument [14] and provide a first demonstration of its use as a local viscosity probe for water under pressures that go up to $1.3 \mathrm{GPa}$ (above this pressure, the water crystallized). It is important to note that previous attempts to accurately measure viscosity of liquids in a DAC used a rolling ball technique [15-17] where viscosity is inferred from the motions of a ball rolling over the surface of the diamond under the action of gravity or centrifugal forces. Although absolute viscosity determinations are possible in principle, they require the solution of a complex hydrodynamic problem that depends critically on the ball-wall separation and on unknown friction coefficients. For these reasons, in practice, one always relies on scaling laws and measures the relative viscosities with respect to known ambient values. Morevover, high precision measurements with a rolling ball also require a priori knowledge of the density of the sample in the investigated pressure range. Other approaches make use of specifically designed high pressure rheometers (concentric cylinders [18] and oscillating disks [19]) that can rarely reach GPa pressures and again provide only relative viscosity measurement. In this context, our technique has the unique feature of providing absolute viscosity measurements that require no a priori knowledge of sample parameters other than the probe bead's radius. Our silica probe beads are sufficiently hard (bulk modulus of around $40 \mathrm{GPa}$ [20]) that they do not compress noticeably at the pressures we are using.

Optical trapping system.-Counterpropagating optical traps use two focused laser beams, with the foci slightly displaced in the axial direction, to trap objects between the foci. A simple ray optical model is sufficient to describe trapping in the lateral direction: if a spherical object is moved off axis, light is refracted in that direction by the object. The change in the light's direction requires a force to be applied and the reaction force to this on the bead causes it to move back towards the optical axis. Axial confinement is given by light scattered from the bead: if it gets closer to one focus than the other, more light is scattered from the beam forming that focus. This means that the particle experiences more radiation pressure from that beam and is pushed back to the midpoint of the two foci.

Holographic counterpropagating optical traps use a mirror to reflect the laser back towards the trapping site as shown in Fig. 1. The obstacle to implementing this in a diamond anvil cell lies in the geometry of the cell: a mirror placed above the DAC (or even on the top face of the upper diamond) is too far away, as the backwards propagating beam would have to be defocused by several millimeters.
This would make it too large to fit through the aperture in the metal gasket that seals the sample between the diamonds in the focal plane. Coating the bottom surface of the upper diamond might offer a solution to this; however, the difficulty of coating the tip of the diamond, the near certainty of damaging said coating during the assembly and use of the cell, and direct exposure to sample fluids make this impractical. To avoid these difficulties, we employ a reimaging system to place a virtual mirror inside the upper diamond, using an assembly with two lenses and a dielectric mirror that fits between the sample and the microscope condenser. Our optical system is detailed in Fig. 2.

Reimaging the mirror is not ideal, as the double-pass through the optics and the upper diamond anvil reduces both the intensity and numerical aperture of the backpropagating beam. We, therefore, modify the design of the hologram from that used in previous work [13] to form the two foci from distinct annular regions of the SLM as shown in Fig. 1. The forward propagating part of the beam is formed using the outer part of the SLM, i.e., the highest NA part of the beam. Using an annular aperture to cut out the center of the beam reduces the "scattering" component of the forward beam [21]. This is important, as it reduces the amount of power required in the backwardspropagating beam (which is unavoidably attenuated by the virtual mirror). The back-propagating beam is formed using the region inside the annulus that forms the forward beam, so that no light is cut off by the reduced NA of the mirror reimaging optics. Finally, the very center of the SLM blocks the beam, to prevent the particle being pushed out of the trap by out-of-focus light from the backpropagating beam.

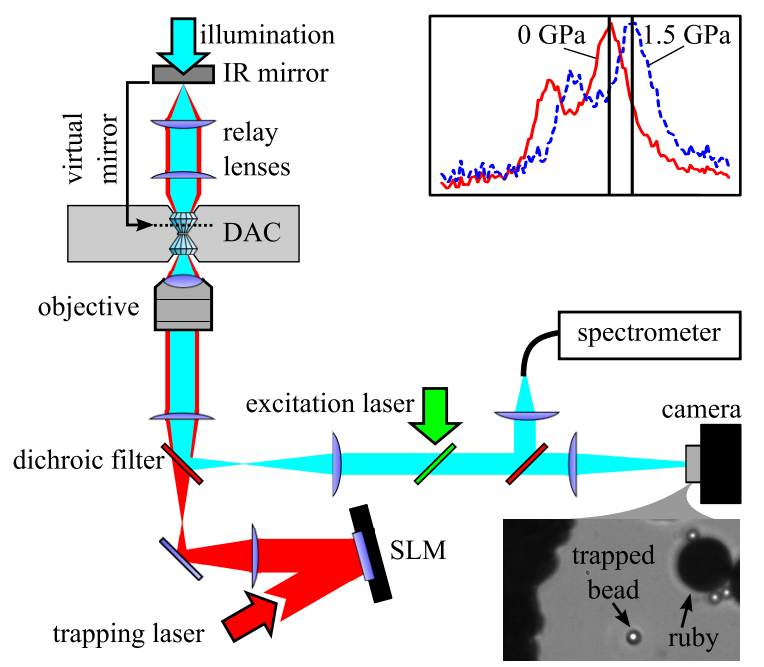

FIG. 2 (color online). The optical system used to trap at high pressure. The trapping laser is controlled using a liquid crystal spatial light modulator as described in Ref. [14], but a $10 \times$, 0.3 NA objective is used. The inset shows the $R 1$ line as recorded at atmospheric pressure and $1.5 \mathrm{GPa}$ and the fitted peak values. 
Some earlier implementations of counterpropagating traps used feedback control to stabilize the trap [22,23]; however, the design described here is stable without feedback control. Removing the requirement for feedback control avoids any distortion of the viscosity measurement due to trap motion and also removes the need to accurately measure the particle's axial position. Stable axial trapping does, however, require careful adjustment of the power in the two beams. The ratio of powers can be adjusted by changing the radius of the inner edge of the annulus forming the forwards-propagating beam.

Pressure control.-In this Letter, we used a diamond anvil cell optimized for light microscopy, $\mu$ Scope DAC RT-G from EasyLab technologies. This provided a working distance of $8 \mathrm{~mm}$ on both sides, in a symmetric package $16 \mathrm{~mm}$ thick that was held on an automated translation stage. Pressure was applied to the outer faces of the diamonds via a gas membrane, which could be pressurized up to $20 \mathrm{MPa}$; a pressure controller was used to increase the applied pressure in controlled steps, so that many data points could be taken in a single compression cycle.

As the relationship between applied pressure and pressure in the cell is not constant or linear, it was measured spectroscopically using ruby beads [24]. This was achieved by adding a dichroic filter to the imaging arm of the tweezers system to couple in a $15 \mathrm{~mW}, 532 \mathrm{~nm}$ laser module. This was focused into a ruby sphere $20-30 \mu \mathrm{m}$ in diameter and the fluorescence was coupled into an Ocean Optics HR4000 spectrometer via a multimode fiber. A second dichroic beam splitter allowed the camera to be used simultaneously with the spectrometer. The $R 1$ fluorescence peak (694 $\mathrm{nm}$ at atmospheric pressure) was tracked using quadratic curve fitting, allowing us to follow it more accurately than the $0.05 \mathrm{~nm}$ pixel pitch of the spectrometer. We convert the wavelength into pressure using the formula given by Ref. [25], after calibrating the spectrometer with the measured value at atmospheric pressure. Example spectra are shown in an inset of Fig. 2.

Viscosity measurement.-An optically trapped particle in water still exhibits Brownian motion, although its diffusion is curtailed on longer time scales by the springlike optical trap. This is quantified by the power spectrum of the motion, which has a characteristic Lorentzian shape [26]:

$$
\mathrm{PSD}=\frac{D}{2 \pi^{2}} \frac{1}{f^{2}+f_{c}^{2}}
$$

where $f_{c}$ is the corner frequency (related to trap strength) and $D$ is the diffusion coefficient. Plotting the power spectrum, as done in Fig. 3, shows distinct regions: a plateau at low frequency corresponding to trapping and a high frequency tail that falls off as $D / 2 \pi^{2} f^{2}$ corresponding to free diffusion. The gradient of the high-frequency tail with respect to $f^{-2}$ allows us to recover the diffusion coefficient of the bead that is related to the viscosity $\eta$ through the Stokes-Einstein relation:

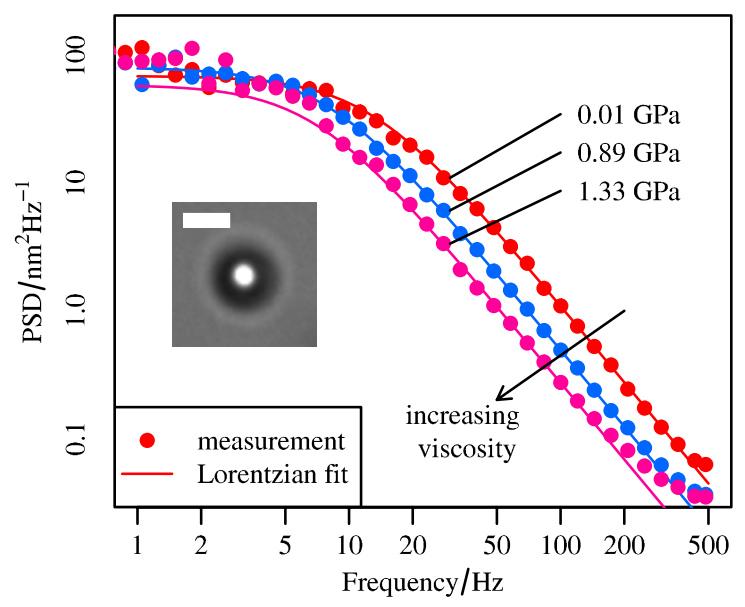

FIG. 3 (color online). Power spectral density of a trapped bead's motion at different pressures (points) with Lorentzian fits (lines). Note the shift of the high-frequency tail, which allows us to determine viscosity. Inset: image of a bead, from which its position was determined at $1 \mathrm{kHz}$ (scale bar: $5 \mu \mathrm{m}$ ).

$$
D=\frac{k_{B} T}{6 \pi \eta a}
$$

where $T$ is absolute temperature, $k_{B}$ is Boltzmann's constant, and $a$ is particle radius. Indeed, by following the motion of trapped beads in both static and moving optical traps, one can recover frequency-dependent viscoelastic moduli of the trapping medium [27-29]. This analysis can also be extended to multiple directions, allowing us to measure anisotropic viscous and viscoelastic properties; in most cases, the equation of motion is separable into three orthogonal directions. Digital holographic tracking of the bead would allow us to recover 3D particle motion and hence, probe material properties in 3D [30]. The same technique can also measure the bead's radius with a precision of $0.1 \%$, eliminating the calibration uncertainty due to polydispersity of the beads and allowing us to quantify any change in the bead's size due to pressure [31].

Results.-The assembled sample cell, shown in the inset of Fig. 2, contains the material to be probed (in this case water) along with $5 \mu \mathrm{m}$ diameter silica microspheres (Bangs Labs) and a ruby sphere for pressure measurement. The anvil cell was placed on the microscope, the gasket aperture was located, and a particle trapped as described previously. The particle's motion was followed on a high speed video image at $1 \mathrm{kHz}$ using a DALSA Genie HM640 camera and image analysis routines in LABVIEW and $\mathrm{C}$. The cell was gradually compressed by applying pressure to the gas membrane and the pressure in the cell was monitored by tracking the $R 1$ fluorescence peak. The pressure was allowed to stabilize for a few minutes after each change to allow for relaxation of the steel gasket. Below its crystallisation pressure, working in a liquid ensures that the pressure is constant across the cell. 


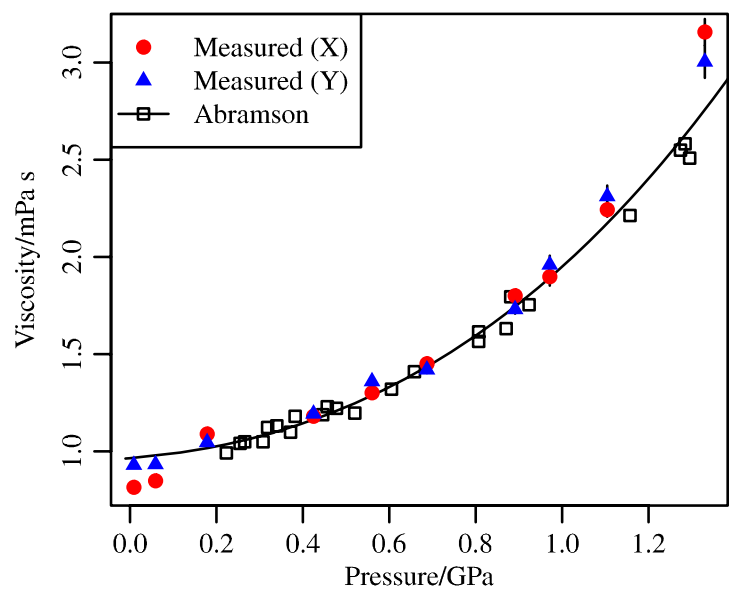

FIG. 4 (color online). Viscosity measured using particle tracking as a function of pressure. The data and polynomial fit presented by Abramson using a rolling platinum sphere are shown for comparison [17].

For each pressure in the cell, we recorded $10^{5}$ particle positions, corresponding to around $100 \mathrm{~s}$. From this data, the power spectrum of the particle's motion and thus, the viscosity was calculated. It was also possible to move the trap periodically and observe the bead's response, which can provide better signal-to-noise in more viscous materials by allowing us to observe the creep response of the fluid. Figure 4 shows the viscosity measured over a range of pressures up to the crystallization point, along with the accepted data measured by Abramson at $21^{\circ} \mathrm{C}$ [17]. The two data sets agree well up to $1 \mathrm{GPa}$ and start deviating in the metastable region above it. Viscosity measured in the $x$ and $y$ directions is the same, as one would expect for an isotropic medium such as water. However, when combined with $z$ position measurement, the ability to probe anisotropy in the liquid's properties could be very useful in verifying whether the system is still in hydrostatic equilibrium.

Conclusion.-We have demonstrated optical trapping inside a diamond anvil cell at high pressure, providing the hitherto unavailable ability to move and trap particles in the high pressure medium. This was all performed using a portable optical trapping instrument to facilitate the integration of this technique into high pressure experiments outside of the laser laboratory. We have used this in conjunction with high speed particle tracking microscopy to measure absolute viscosity as a function of pressure. Once calibrated, optically trapped beads can be used as force transducers, for example, to measure the change in mechanical properties of cell membranes at high pressure. This technique can make possible a wide range of new mechanical and rheological experiments in high pressure physics and biology.

R.W.B. is supported by a Research Fellowship at Queens' College, Cambridge. M.J.P. acknowledges financial support from the Royal Society. This work was supported by EPSRC. R.D.L. and F.S. acknowledge funding from IIT-SEED BACTMOBIL Project and MIUR-FIRB Project No. RBFR08WDBE.

*Present address: Department of Physics, University of Cambridge, Cavendish Laboratory, J.J. Thomson Avenue, Cambridge CB3 OHE, United Kingdom. richard.bowman@cantab.net ${ }^{\dagger}$ http://www.physics.gla.ac.uk/Optics/

[1] P. W. Bridgman, J. Biol. Chem. 19, 511 (1914).

[2] L. Dubrovinsky, N. Dubrovinskaia, V. Prakapenka, and A. Abakumov, Nat. Commun. 3, 1163 (2012).

[3] R. T. Howie, C. L. Guillaume, T. Scheler, A. F. Goncharov, and E. Gregoryanz, Phys. Rev. Lett. 108, 125501 (2012).

[4] G. Piermarini, R. Forman, and S. Block, Rev. Sci. Instrum. 49, 1061 (1978).

[5] A. Ashkin, J. M. Dziedzic, J.E. Bjorkholm, and S. Chu, Opt. Lett. 11, 288 (1986).

[6] A. Ashkin, Phys. Rev. Lett. 24, 156 (1970).

[7] P. Rodrigo, V. R. Daria, and J. Glückstad, Appl. Phys. Lett. 86, 074103 (2005).

[8] G. Sinclair, P. Jordan, J. Leach, M. Padgett, and J. M. Cooper, J. Mod. Opt. 51, 409 (2004).

[9] M. Pitzek, R. Steiger, G. Thalhammer, S. Bernet, and M. Ritsch-Marte, Opt. Express 17, 19414 (2009).

[10] S. Zwick, T. Haist, Y. Miyamoto, L. He, M. Warber, A. Hermerschmidt, and W. Osten, J. Opt. A 11, 034011 (2009).

[11] M. Reicherter, T. Haist, E. Wagemann, and H. J. Tiziani, Opt. Lett. 24, 608 (1999).

[12] D. G. Grier, Nature (London) 424, 810 (2003).

[13] S. B. G. Thalhammer, R. Steiger, and M. Ritsch-Marte, J. Opt. 13, 044024 (2011).

[14] G. M. Gibson, R. W. Bowman, A. Linnenberger, M. Dienerowitz, D. B. Phillips, D. M. Carberry, M. J. Miles, and M. J. Padgett, Rev. Sci. Instrum. 83, 113107 (2012).

[15] H. King, E. Herbolzheimer, and R. Cook, J. Appl. Phys. 71, 2071 (1992).

[16] R. Cook, C. Herbst, and H. King, Jr., J. Phys. Chem. 97, 2355 (1993).

[17] E. H. Abramson, Phys. Rev. E 76, 051203 (2007).

[18] S. Bair, M. Khonsari, and W. O. Winer, Tribol. Int. 31, 573 (1998).

[19] K. Dudziak and E. Franck, Ber. Bunsen-Ges. Phys. Chem. 70, 1120 (1966).

[20] G. W. C. Kaye and T.H. Laby, Tables of Physical and Chemical Constants, Kaye and Laby Online, Version 1.0, Elasticities and Strengths (2005), p. 2.2.2, http://www .kayelaby.npl.co.uk/general_physics/2_2/2_2_2.html.

[21] R. W. Bowman, G. Gibson, and M. Padgett, Opt. Express 18, 11785 (2010).

[22] S. Tauro, A. Bañas, D. Palima, and J. Glückstad, Opt. Express 18, 18217 (2010).

[23] R. W. Bowman, G. Thalhammer, A. Jesacher, G. Gibson, M. Ritsch-Marte, and M. J. Padgett, Opt. Express 19, 9908 (2011). 
[24] R. Forman, S. Block, J. Barnett, and G. Piermarini, Science 176, 284 (1972).

[25] A. Chijioke, W. Nellis, A. Soldatov, and I. Silvera, J. Appl. Phys. 98, 114905 (2005).

[26] K. Berg-Sørensen and H. Flyvbjerg, Rev. Sci. Instrum. 75, 594 (2004).

[27] A. Yao, M. Tassieri, M. Padgett, and J. Cooper, Lab Chip 9, 2568 (2009).
[28] M. Tassieri, G. M. Gibson, R. M. L. Evans, A. M. Yao, R. Warren, M. J. Padgett, and J. M. Cooper, Phys. Rev. E 81, 026308 (2010).

[29] A. I. Bishop, T. A. Nieminen, N. R. Heckenberg, and H. Rubinsztein-Dunlop, Phys. Rev. Lett. 92, 198104 (2004).

[30] G. Bolognesi, S. Bianchi, and R. Di Leonardo, Opt. Express 19, 19245 (2011).

[31] K. Xiao and D. G. Grier, Phys. Rev. Lett. 104, 028302 (2010). 